\title{
Capparis; A Candidate for Therapy in Patients with Hepatopulmonary Syndrome
}

\author{
Saeed Sardari ${ }^{1}$, Faramarz Fallahi ${ }^{2}$, Fatemeh Emadi ${ }^{1}$, Narjes Khavasi ${ }^{3}$ and Seied Saeid Esmaeili ${ }^{1,{ }^{*}}$ \\ ${ }^{1}$ Department of Iranian Traditional Medicine, Faculty of Medicine, Shahed University, Tehran, Iran \\ ${ }^{2}$ Department of Cardiology Mostafa Khomeini Hospital, Faculty of Medicine, Shahed University, Tehran, Iran \\ ${ }^{3}$ Department of Iranian Traditional medicine, School of Medicine, Zanjan University of Medical Sciences, Zanjan, Iran \\ Corresponding author: Assistant Professor, Department of Iranian Traditional Medicine, Faculty of Medicine, Shahed University, No 17, Salamat Clinic (Iranian Traditional \\ Medicine), Tehran, Iran. Tell: +98-9126223925, Fax: +98-2122882521, Email: dr.esmaeili@chmail.ir
}

Received 2018 February 04; Revised 2018 April 17; Accepted 2018 July 21.

Keywords: Capparis spinosa, Hepatopulmonary Syndrome, Iranian Traditional Medicine

\section{Dear Editor,}

Hepatopulmonary syndrome (HPS) is commonly a pulmonary manifestation described as the triad of liver disease, arterial hypoxia, and intra-pulmonary vasodilatation. Dyspnea and cyanosis are the most common complaints of patients with HPS (1). The pathogenesis of HPS is not well known, but the treatment procedure is based on four pathophysiological mechanisms including inactivation of endothelin-1 (ET-1), inhibition of pulmonary angiogenesis, blockage of nitric oxide synthesis, and inhibition of bacterial translocation. Currently, liver transplantation is the only certain treatment of HPS (2).

One of the physicians of Iranian traditional medicine (ITM), Saleh Nasolah Halabi, characterized the disease (without mentioning the name) by stiffness of the left side of the abdomen, splenomegaly, weight loss, body weakness, cyanosis, dyspepsia, shortness of breath, edema, and wounds in the legs. He believed that it is lethal; symptoms, processes, and prognosis are similar to those of HPS. His suggested treatment in the early stages of the disease is the application of Caparis spinosa and its products (2).

Previous researches pointed out that pulmonary vascular angiogenesis can be inhibited by Quercetin, due to involvement in Akt/NF- $\kappa$ B and VEGFA/VEGFR-2 pathways (3). Moreover, Quercetin antioxidant activity and its ability to modulate the TLR2/TLR4 and MAPK/NF- $\kappa$ B signaling pathways inhibit CCl4-induced inflammation in rat (4) similar to some medicinal plants such as C. spinosa (5).

Caper (C. spinosa) has pharmaceutical properties due to a rich source of flavonoids including Quercetin, kaempferol, and anthocyanins. C. spinosa has hepatoprotective, antioxidant, and anti-inflammatory effects.
Furthermore, it has a positive effect on organs such as the lung, heart, liver, and spleen $(6,7)$. Previously undertaken clinical trials suggest that $C$. spinosa can have positive effects on liver regeneration (8).

According to ITM, some diseases in an organ may occur due to complications in another organ without any clear communication. Spleen and pancreas are the main sources of liver diseases. Splenomegaly leads to a decrease in the liver span and body weight. C. spinosa may have beneficial effects on liver through improvement of spleen function (9). In a recent study, the relationship between spleen, metabolic disorders, liver (as liver spleen axes) cirrhosis, splenomegaly, and HPS is reported (10-12).

A limitation of this viewpoint is the attitude of ITM towards the application of such medications in the early stages of the disease (2).

Carrying out of animal model and also in vitro studies at an early stage of the hepatopulmonary syndrome with the application of $C$. spinosa can be helpful merely via meticulous consideration of the process.

\section{References}

1. Mohammad Alizadeh AH, Fatemi SR, Mirzaee V, Khoshbaten M, Talebipour B, Sharifian A, et al. Clinical features of hepatopulmonary syndrome in cirrhotic patients. World $J$ Gastroenterol. 2006;12(12):1954-6. [PubMed: 16610006]. [PubMed Central: PMC4087525].

2. [No Authors Listed]. Gayat ol etghan fi tadbir el badan el ensan [Disease]. Tehran, Iran: Iran university of Medical and Sciences, 17th Century; 2009.

3. Li X, Chen Y, Wang L, Shang G, Zhang C, Zhao Z, et al. Quercetin alleviates pulmonary angiogenesis in a rat model of hepatopulmonary syndrome. Braz J Med Biol Res. 2016;49(7). doi: 10.1590/1414431X20165326. [PubMed: 27383124]. [PubMed Central: PMC4942229]. 
4. Ma JQ, Li Z, Xie WR, Liu CM, Liu SS. Quercetin protects mouse liver against $\mathrm{CCl}(4)$-induced inflammation by the TLR2/4 and MAPK/NF-kappaB pathway. Int Immunopharmacol. 2015;28(1):531-9. doi: 10.1016/j.intimp.2015.06.036. [PubMed: 26218279].

5. Tlili N, Feriani A, Saadoui E, Nasri N, Khaldi A. Capparis spinosa leaves extract: Source of bioantioxidants with nephroprotective and hepatoprotective effects. Biomed Pharmacother. 2017;87:171-9. doi: 10.1016/j.biopha.2016.12.052. [PubMed: 28056421].

6. Eshraghian A, Kamyab AA, Yoon SK. Pharmacological treatment for hepatopulmonary syndrome. Biomed Res Int. 2013;2013:670139. doi: 10.1155/2013/670139. [PubMed: 24102057]. [PubMed Central: PMC3786536].

7. Moufid A, Farid O, Eddouks M. Pharmacological properties of capparis spinosa linn. Int J Diabetol Vascular Dis Res. 2015:99-104. doi: 10.19070/2328-353x-1500020.

8. Khavasi N, Somi MH, Khadem E, Faramarzi E, Ayati MH, Fazljou SMB, et al. Effect of daily caper fruit pickle consumption on disease regression in patients with non-alcoholic fatty liver disease: A double- blinded randomized clinical trial. Adv Pharm Bull. 2017;7(4):645-50. doi: 10.15171/apb.2017.077. [PubMed: 29399555]. [PubMed Central: PMC5788220].

9. Aveccena. Qanoon fi teb [Fundamentals of medicines - diseases - pharmaceuticals and industry]. Beirut: Dar ol Torath ol Arabieh, 11th Century; 2005.

10. Robinette CD, Fraumeni JF. Splenectomy and subsequent mortality in veterans of the 1939-45 war. Lancet. 1977;2(8029):127-9. doi 10.1016/S0140-6736(77)90132-5. [PubMed: 69206].

11. Tarantino G, Scalera A, Finelli C. Liver-spleen axis: intersection between immunity, infections and metabolism. World J Gastroenterol. 2013;19(23):3534-42. doi: 10.3748/wjg.v19.i23.3534. [PubMed: 23801854]. [PubMed Central: PMC3691032].

12. Emtiazy M, Choopani R, Khodadoost M, Tansaz M, Nazem E. Atheroprotector role of the spleen based on the teaching of Avicenna (Ibn Sina). Int J Cardiol. 2013;167(1):26-8. doi: 10.1016/j.ijcard.2012.06.020. [PubMed: 22726399]. 